\title{
Micromechanical testing of oxidized grain boundaries in Nickel alloys from nuclear reactors
}

Sergio Lozano-Perez ${ }^{1}$, Helen Dugdale ${ }^{1}$, David E J Armstrong ${ }^{1}$, Takumi Terachi ${ }^{2}$, Takuyo Yamada $^{2}$, Edmund Tarleton ${ }^{1}$ and Steve G Roberts ${ }^{1}$

${ }^{1}$ Department of Materials, University of Oxford, Oxford OX1 3PH, United Kingdom.

${ }^{2}$ INSS, 64 Sata, Mihama-cho, Mikata-gun, Fukui 919-1205, Japan.

\begin{abstract}
The fracture behaviour of individual grain boundaries has been studied in order to understand the mechanisms controlling stress corrosion cracking in nuclear reactors. In particular, the role of oxidation in facilitating crack initiation and propagation has been reviewed. Nickel alloys from pressurized water reactors (PWRs) have been tested in simulated primary water conditions to induce grain boundary oxidation. Microcantilevers containing an oxidized grain boundary plane have been prepared and tested for fracture. The brittle nature of the oxide was demonstrated and the required stress to fracture measured.
\end{abstract}

\section{INTRODUCTION}

Stress corrosion cracking (SCC) has been a concern for the nuclear industry (amongst others) for decades. At present, we lack a clear understanding of the underlying mechanisms and several have been proposed. Some of the most accepted mechanisms rely on the fracture of a brittle phase (e.g. oxide in a grain boundary). The Selective Internal Oxidation (SIO) was proposed by Scott and LeCalvar [1] after analyzing a number of thermodynamic and kinetic features of the cracking and expressing 'the difficulties in reconciling those trends with the anodic dissolution or hydrogen embrittlement mechanistic hypothesis for cracking'. The model is based on two relatively simple observations: that Alloy 600 is known to oxidize internally along grain boundaries in the presence of primary water and that the failure of the alloy is intergranular. The model supposes that the $\mathrm{Cr}$-rich oxide present along the grain boundary is brittle, and therefore causes embrittlement of the grain boundary. This then provides an easy crack pathway through the alloy, causing it to fail along the grain boundaries. The fracture occurs in stages, with oxygen diffusing down a section of grain boundary, thus embrittling it before a crack grows down that particular section. Once the crack has opened up, oxygen diffuses further into the alloy, down a deeper section of grain boundary, which then also cracks. Although the idea is plausible and some evidence of oxides ahead of crack tips [2] has been reported, there is no quantitative data on the brittleness of these oxides or what exact role they play in the crack propagation. The aim of the paper is to investigate the SIO mechanism and determine the viability of its explanation for the behavior of Alloy 600 in PWR primary water. In order to do this, a novel technique to investigate the mechanical properties of the grain boundary oxide was developed in order to determine whether the oxide did indeed have an embrittling 
effect on the grain boundary. The technique relies on the controlled fracture of cantilevers containing an oxidized grain boundary.

\section{EXPERIMENT}

The Alloy 600 coupon was provided after the autoclave testing by the Institute of Nuclear Safety Systems (INSS), Japan. The chemical composition was 0.017 C, 0.32 Mn, 8.57 Fe, 74.03 $\mathrm{Ni}$ and $16.21 \mathrm{Cr}$ (all in wt.\%). The coupon was mill annealed at $1050^{\circ} \mathrm{C}$ for 15 minutes after fabrication. The corrosion test was performed in an autoclave under simulated PWR primary water conditions $(2.75 \mathrm{ppm} \mathrm{DH} 2,500 \mathrm{ppm} \mathrm{B}$ and $2 \mathrm{ppm} \mathrm{Li})$ at $360^{\circ} \mathrm{C}$ for $2700 \mathrm{~h}$.

After the $2700 \mathrm{~h}$ in simulated PWR primary water, oxides on the sample surfaces were observed, as well as the selective oxidation of grain boundaries. Prior TEM characterization had revealed that intergranular oxidation could reach depths over $5 \mu \mathrm{m}$ [3]. A Zeis NVision 40 focussed ion beam (FIB)- scanning electron microscope (SEM) was used for the preparation and imaging of the cantilevers. Once suitable grain boundaries had been identified, cantilevers were milled across the grain boundary by FIB, prepared as described by [4].
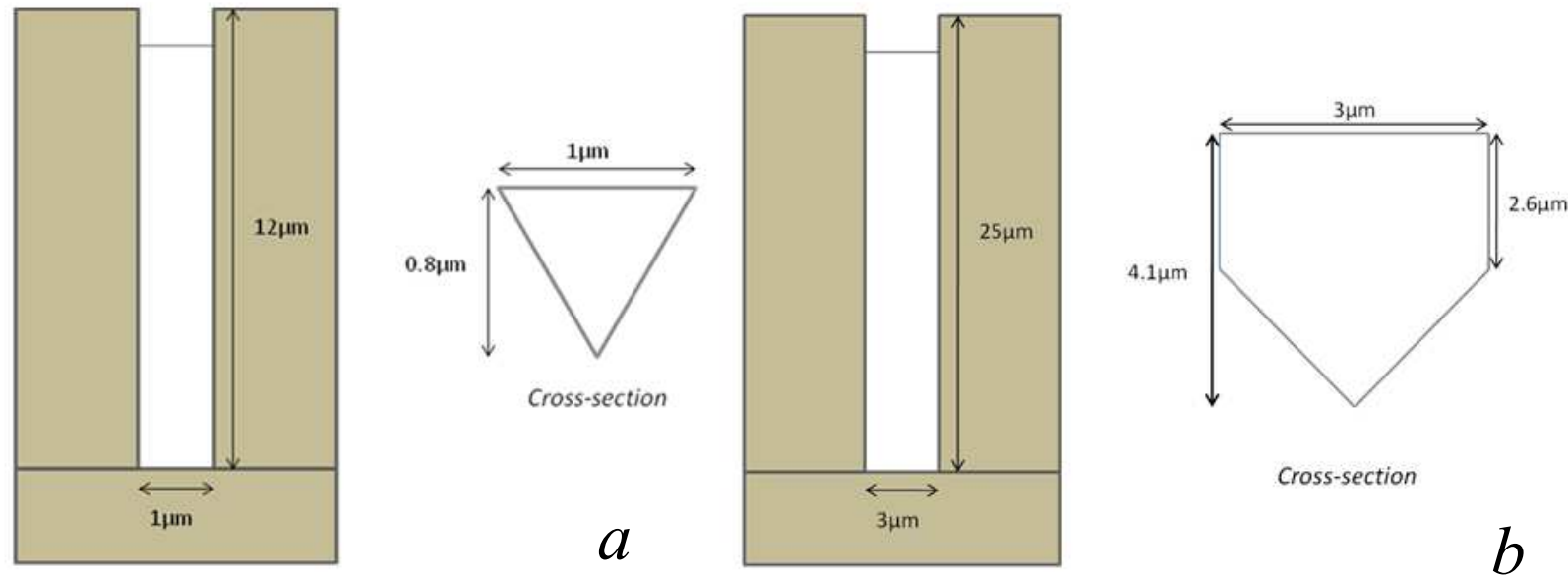

Cross-section

$b$
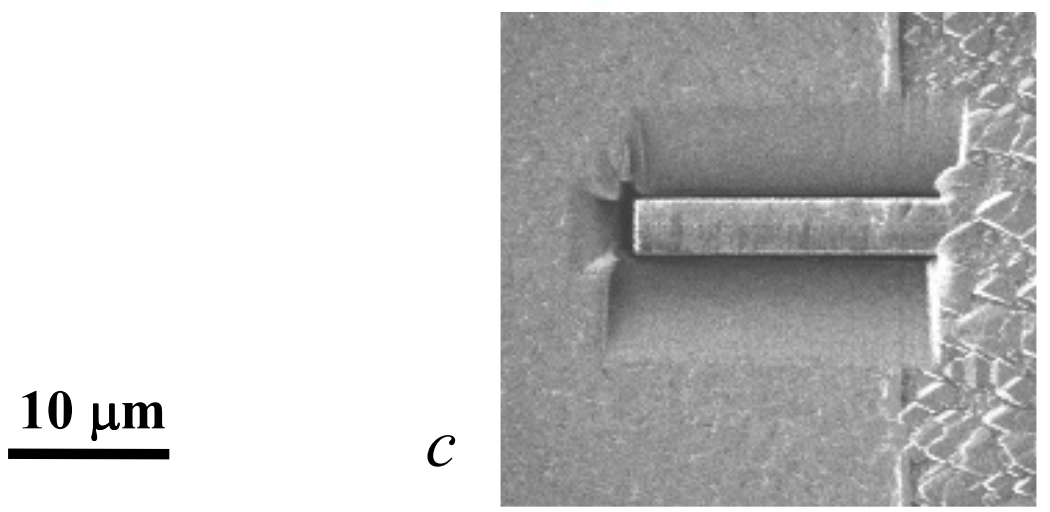

Figure 1: Cantilever Designs, (a) Type 'a': triangular cross-section and (b) Type 'b': pentagonal cross-section; (c) FIB SE image showing a type b cantilever milled with its base containing an oxidized grain boundary 
In order to account for the differing oxide penetration depths along the grain boundary planes, two different cantilever designs were chosen, as shown in Figure 1, which will be referred as type 'a' (triangular) (Figure 1a) and type 'b' (pentagonal) (Figure 1b). An example of a type $b$ cantilever can be seen in Figure 1c. The grain boundary runs vertically on the image and it is easy to spot due to the different oxidation rates experienced by the grain on the left and the grain on the right. In addition, since the grain boundary oxidized preferentially, a thicker outer layer of oxide grown on its intersection with the exposed surface can be also used as a marker.

After preparation, the cantilevers were tested in a MTS Nanoindenter XP, using a diamond Berkovich tip. The indenter tip was placed at the end of the cantilever and used to displace the cantilever at a constant displacement rate of $5 \mathrm{~nm} / \mathrm{s}$ and a load-displacement curve recorded. In previous work surface profilometry was performed using the NanoVision option in the MTS nanoindenter. This has been used to image the cantilever prior to testing, allowing accurate placement of the tip on the end of the cantilever. In these experiments this was not possible. During scanning it was found that loosely bonded oxide particles on the top surface would be picked up on the end of the indenter tip and the scanning resolution would be insufficient to image the cantilever. Instead the indenter tip was aligned using series of microscope to indenter calibrations at high magnification. This allowed the indenter tip to be aligned with the end of the cantilever for testing, however the success rate of testing was significantly lower than when using the NanoVision imaging method. Once tested, the cantilevers were then imaged in a FIB-SEM (Zeiss NVision), where the FIB 3D slicing technique was used to reconstruct the oxidized grain boundary portion of the cantilever in 3D. More details about this technique can be found in [5]. (S)TEM characterization was performed in a Jeol 3000F equipped with an ultrathin polymer window Oxford Instruments EDX detector and operated at $297 \mathrm{keV}$. EDX mapping using a pixel time of $30 \mathrm{~ms}$ was used to characterize the grain boundary chemistry. A form of multivariate statistical analysis (MSA) consisting of a weighted principal component analysis combined with factor analysis was used to identify the key sources of information in the dataset. Then a "noise-free" dataset was generated, from which all elemental maps shown were obtained. Details of the MSA procedure can be found in [6,7].

\section{RESULTS AND DISCUSSION}

After several cantilevers of both types were tested, the results could be split into three categories: cantilevers exhibiting plastic deformation but no fracture, cantilevers exhibiting brittle fracture along the grain boundary and cantilevers exhibiting crack growth along the grain boundary but no complete fracture. Not all cantilevers tested will be discussed in detail.

\section{$\underline{\text { Plastic Deformation and no fracture }}$}

Many of the cantilevers tested simply deformed plastically during testing and no fracture events could be seen on the load/displacement curves, as shown in Figure 2. They were all type ' $b$ ' (larger cross-section). Evidence of slip was observed on the surface and the cantilevers were plastically deformed and depressed into the trench. 


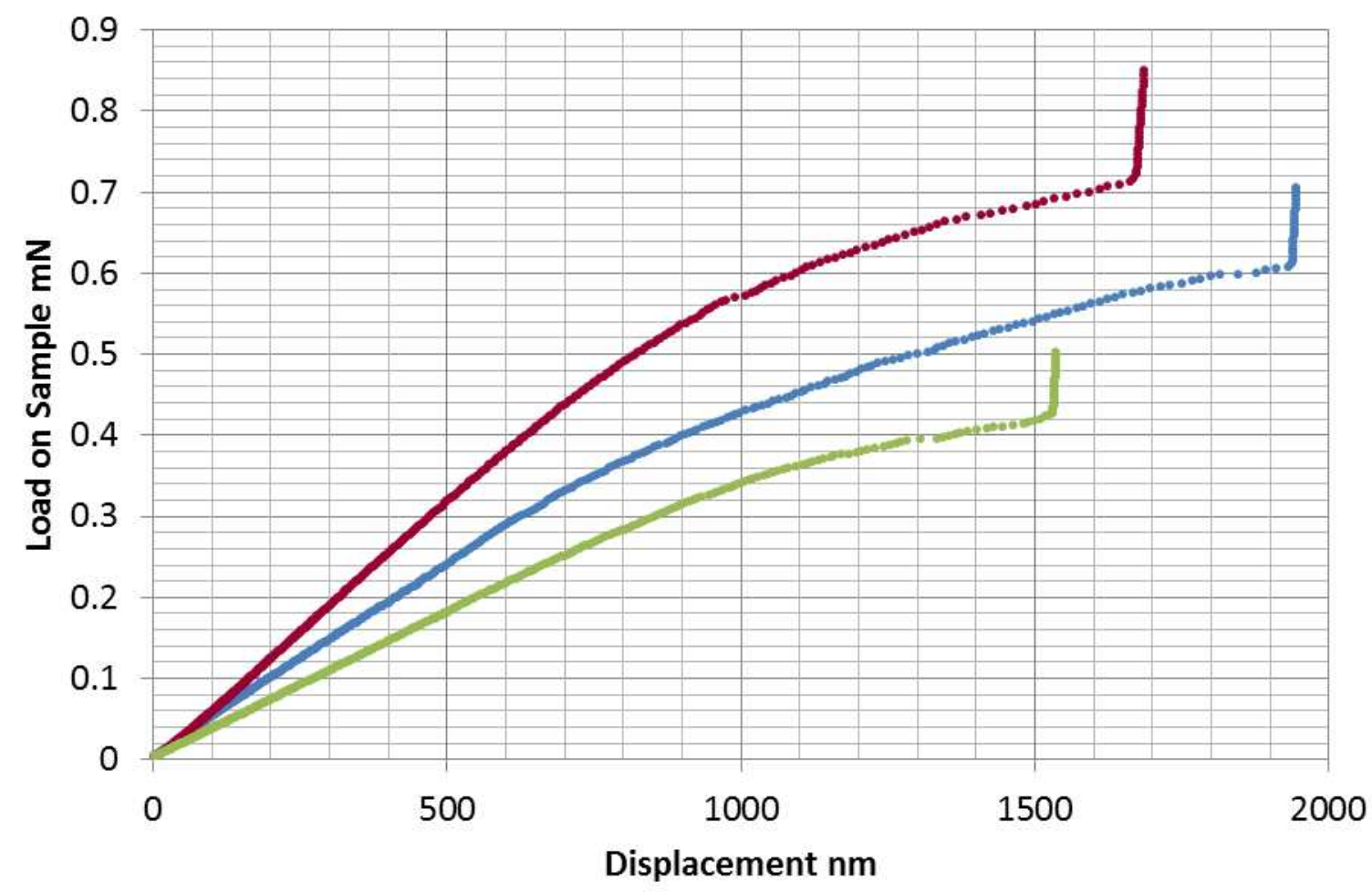

Figure 2: Load displacement curve for some of the type 'b' cantilevers tested which exhibited plastic deformation and no fracture.

\section{Complete fracture}

Most of the type' a' cantilevers tested (smaller cross-section) exhibited complete fracture. Fracture occurred along the oxidized grain boundary, which covered the full cross-section. Although the recorded load-displacement data clearly shows the point where fracture occurred (arrowed in Figure 3), this data was not considered reliable since we believe that the nanoindenter tip contacted the trench edges during testing. This issue has been subsequently fixed and accurate data will be provided in our next publication. In Figure 4, a type a cantilever which exhibited full fracture during testing is shown.

The fact that only "type a" cantilevers exhibited full fracture might be caused by the increasing likelihood of finding intergranular carbides as the size of the cantilever increases. Intergranular carbides alter the oxidation behavior by providing with a sensitized region (low in $\mathrm{Cr}$ ) around carbides, which causes the oxidation to proceed laterally as well as down the grain boundary. This will be seen more clearly in the next section. 


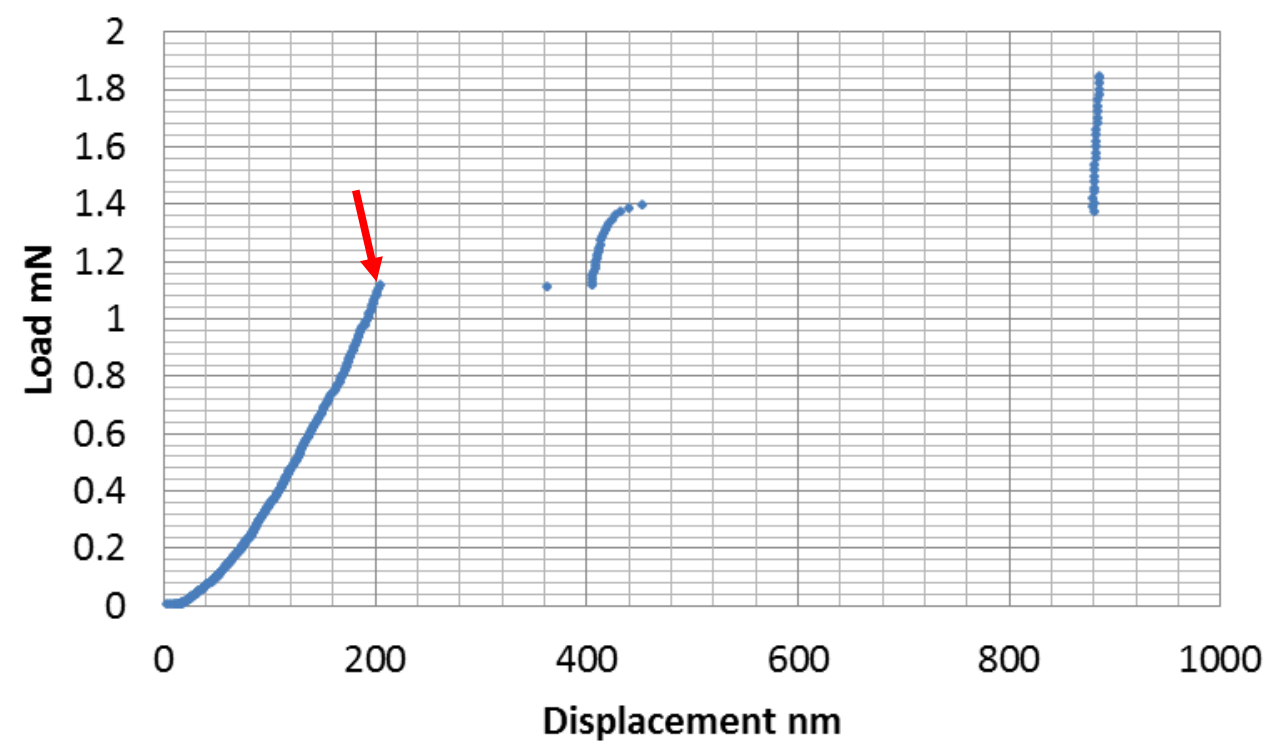

Figure 3: Load displacement curve for the cantilever shown in Figure 4, which exhibited complete fracture. The point where fracture occurred has been arrowed.

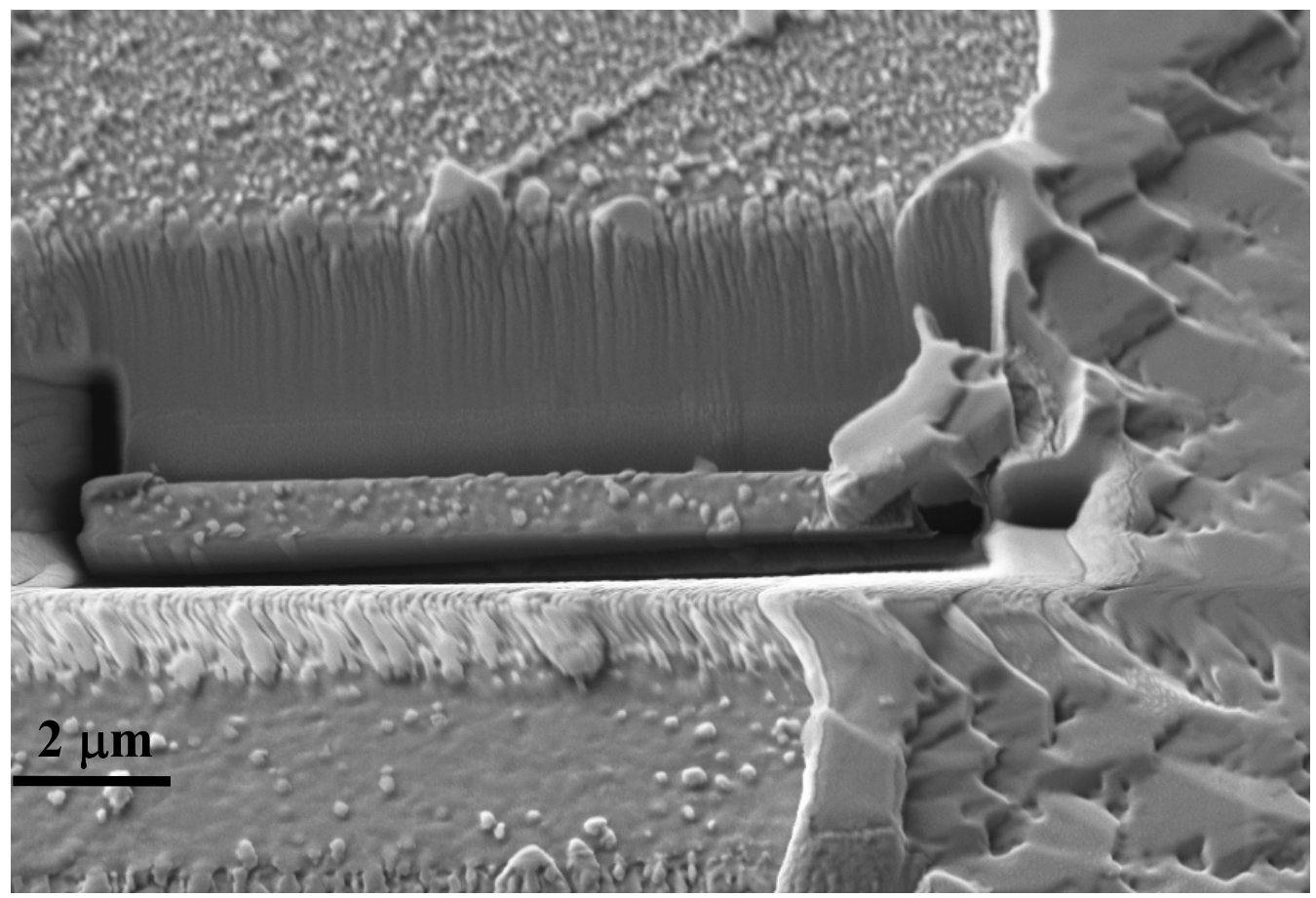

Figure 4: SEM SE image showing a type 'a' cantilever which exhibited complete fracture. The cantilever appears fully detached and is resting at the bottom of the trench. 


\section{Crack growth with incomplete fracture}

Type ' $b$ ' cantilevers, with a depth of over $4 \mu \mathrm{m}$, were usually found to fracture partially, as can be seen in Figure 5. A crack developed during testing but didn't reach the bottom of the sample. A detailed characterization by FIB 3D slicing revealed all the details of the fracture.

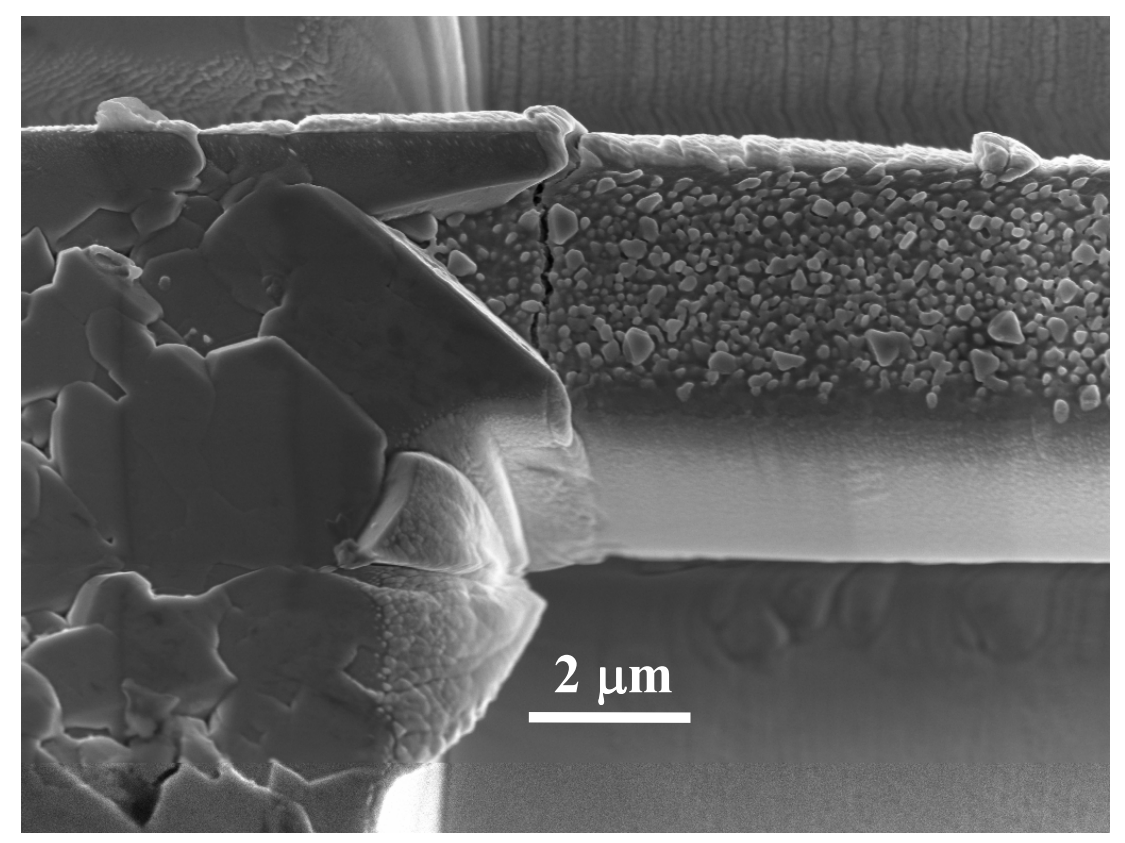

Figure 5: SEM SE image showing a type 'b' cantilever after testing. An intergranular crack has developed, but did not reach the bottom of the sample.

As can be seen in Figure 6, a cross-sectional view of the cantilever reveals that the crack has followed the oxide-metal interface. A detailed examination of the subsequent slices reveals that the Cr-depleted region around the intergranular Cr-rich carbide was oxidized preferentially (see 3D reconstruction in Figure 7). This, however, did not cause the crack to proceed in a straight manner across the oxide and, instead, it circumvented the wider oxide area, always following the oxide-metal interface.

The $3 \mathrm{D}$ reconstruction also reveals that the depth of the crack is not homogeneous along the width of the cantilever, explaining why the sample did not fracture completely: there was not a fully oxidized grain boundary plane across the cantilever. 


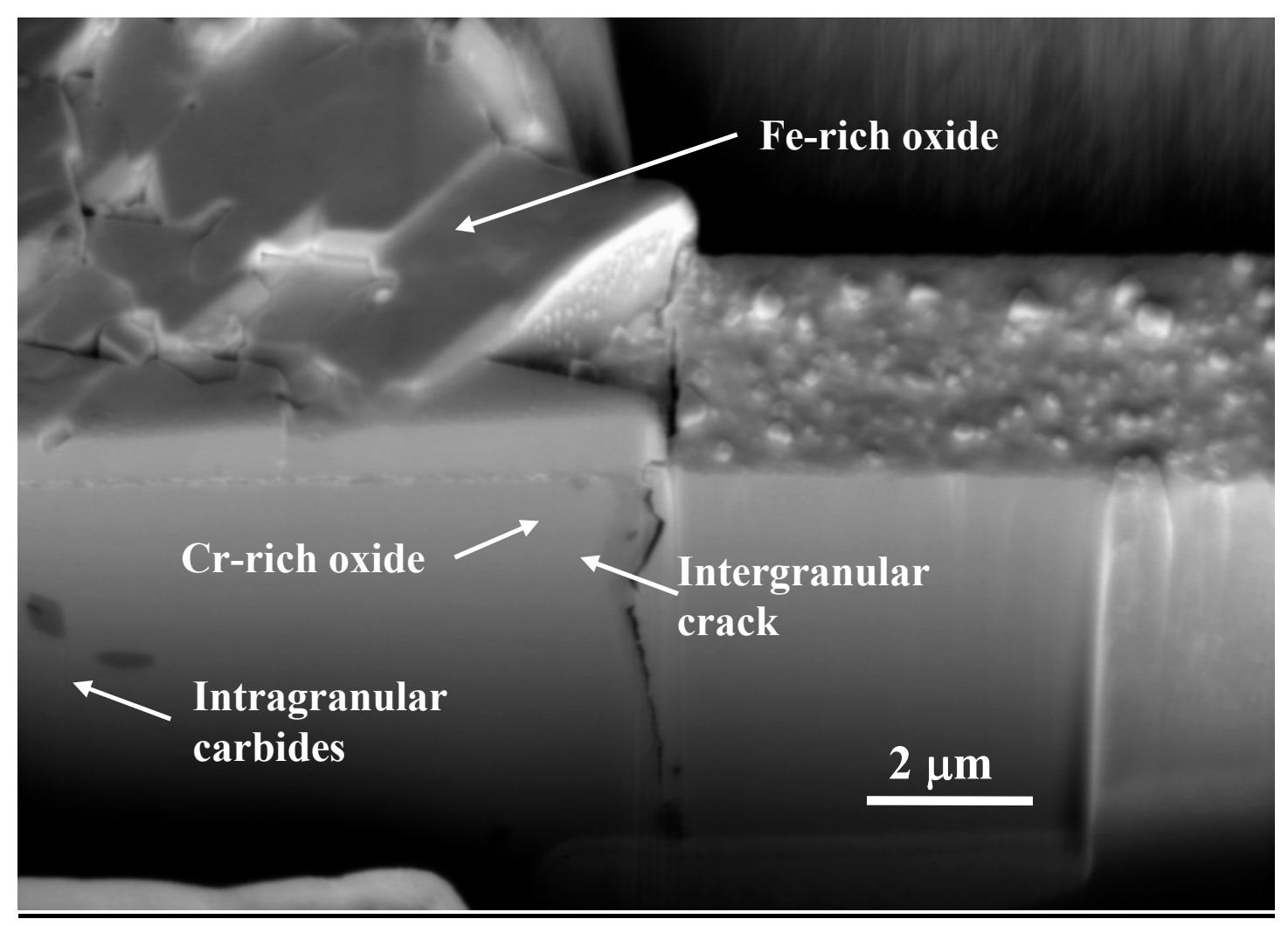

Figure 6: SEM in-lens image showing the same cantilever as in Figure 5 after the first slices have been milled to reveal the internal microstructure. The crack can be seen to propagate following the oxide-metal interface.

The load-displacement curve recorded while testing the cantilever shown in Figure 5 is shown in Figure 8. The point where the crack first appeared is revealed in the plot by the small jumps in the data in the arrowed region. Further analysis is necessary to fully understand this data. At present, finite element modeling of a cantilever with the reconstructed geometry is being performed. A comparison between the experimental and the simulated load-displacement curves will help understanding the fracture behavior and the calculation of the real stress required to fracture the oxide. 


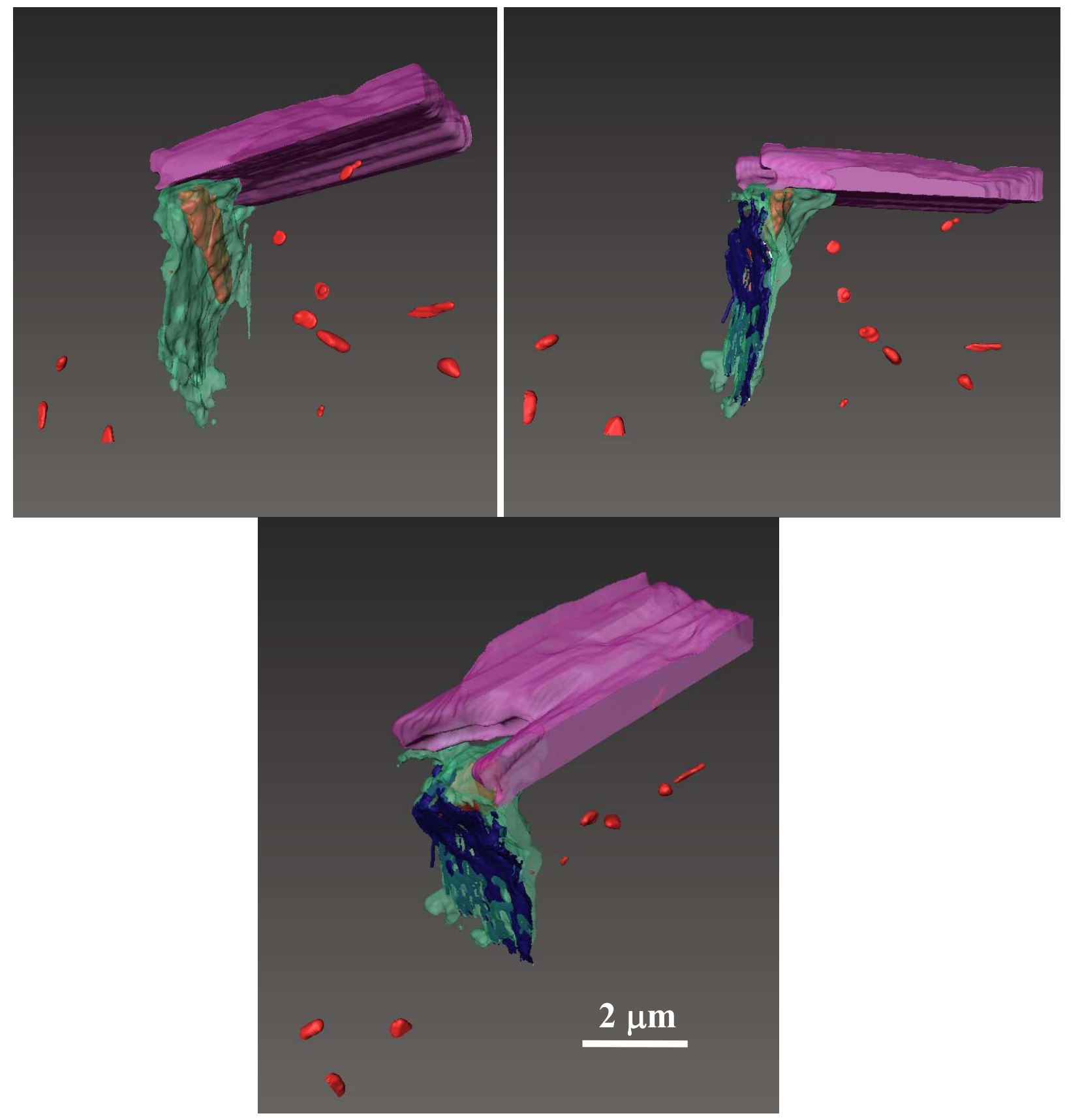

Figure 7: 3D reconstruction of the cantilever shown in Figure 5. 3 different perspectives are shown. Fe-rich oxide is shown in purple, Cr-rich oxide in green, Cr-rich carbides in red and the open crack in blue. 


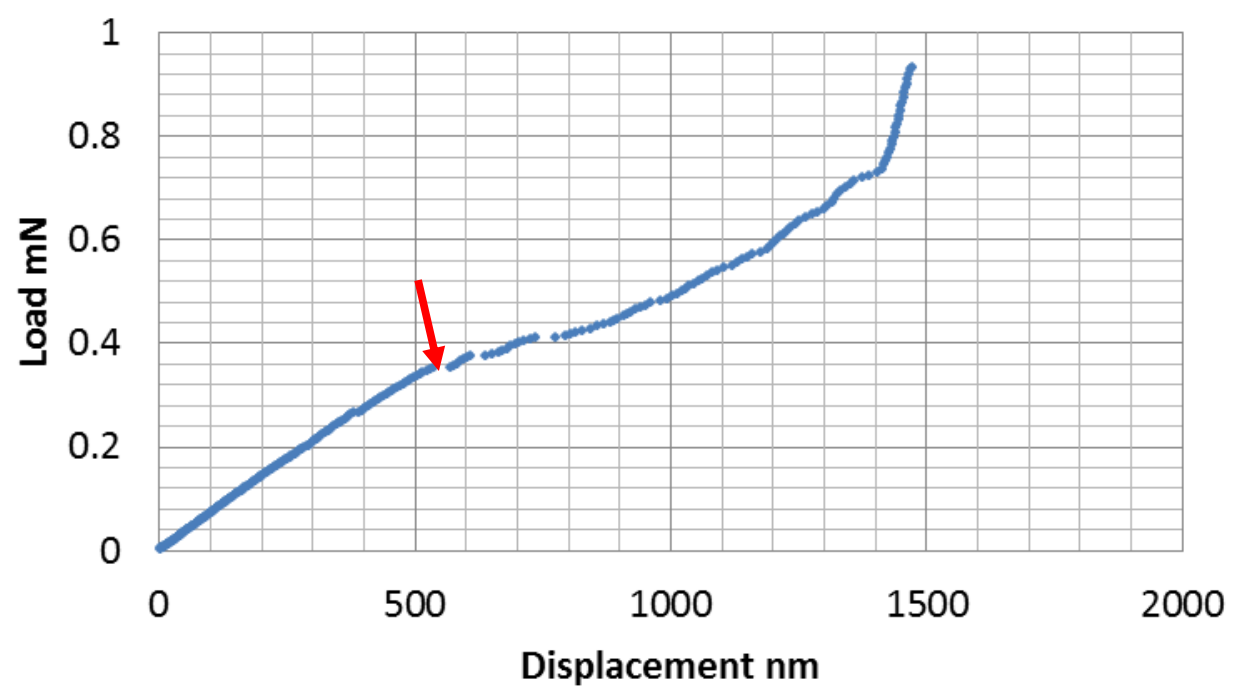

Figure 8: Load displacement curve for the cantilevers shown in Figure 5, which exhibited partial fracture. The point where fracture is believed to initiate has been arrowed.

\section{CONCLUSIONS}

The aim of the paper was to discuss the viability of the Selective Oxidation Model in explaining the stress corrosion cracking behavior of Alloy 600 in the presence of primary water. There are strong indications from the results that the oxide is brittle, suggesting that the SIO model is feasible in principle. In addition, a detailed characterization of the microstructure in the fracture region has revealed a complex crack path which always follows the oxide-metal interface. The grain boundary microstructure is believed to have an important part to play in the response of grain boundaries to stress. In short, three basic observations have been made, all of which support the SIO model:

- $\quad$ Oxidised grain boundaries exhibit brittle fracture events.

- Grain boundaries without internal oxide do not fracture and instead yield.

- Oxidised grain boundaries with carbides can alter the straight crack path along the grain boundary plane, by forcing it to follow the oxide-metal interface, which can be faceted at the Cr-depleted region surrounding the carbides.

These observations however, although do not disprove the SIO model, cannot suggest on their own that grain boundary oxidation is the only mechanism responsible for SCC. Further investigation on the role of Hydrogen and the surrounding microstructure is still required.

\section{ACKNOWLEDGMENTS}

The authors would like to acknowledge the support from INSS (Japan) for the provision and autoclave testing of samples. 


\section{REFERENCES}

1. P.M. Scott and M. Le Calvar, in Proceedings of the Sixth International Symposium on Environmental Degradation of Materials in Nuclear Power Systems - Water Reactors, (NACE, 1993), pp. 657.

2. S. Lozano-Perez, T. Yamada, T. Terachi, M. Schröder, C.A. English, G.D.W. Smith, C.R.M. Grovenor and B.L. Eyre, Acta Materialia 57 5361-5381 (2009).

3. T. Terachi, T. Yamada, K. Arioka and S. Lozano Perez, in Proceedings of the International Symposium on Research for Ageing Management of Light Water Reactors and Its Future Trend edited by: B. Eyre, I. Kimura (INSS, 2007), pp. 215-228.

4. D.E.J. Armstrong, A.J. Wilkinson and S.G. Roberts, Philosophical Magazine Letters 91 394400 (2011).

5. S. Lozano-Perez, K. Kruska, I. Iyengar, T. Terachi and T. Yamada, Corros. Sci. 56 78-85 (2012).

6. P. Trebbia and N. Bonnet, Ultramicroscopy 34 165-178 (1990).

7. S. Lozano-Perez, V. de Castro Bernal and R.J. Nicholls, Ultramicroscopy 109 1217-1228 (2009). 